\title{
An overview of Stackelberg pricing in networks
}

\author{
Citation for published version (APA):
}

van Hoesel, C. P. M. (2006). An overview of Stackelberg pricing in networks. Maastricht University School of Business and Economics. METEOR Research Memorandum No. 043 https://doi.org/10.26481/umamet.2006043

Document status and date:

Published: 01/01/2006

DOI:

10.26481/umamet.2006043

Document Version:

Publisher's PDF, also known as Version of record

\section{Please check the document version of this publication:}

- A submitted manuscript is the version of the article upon submission and before peer-review. There can be important differences between the submitted version and the official published version of record.

People interested in the research are advised to contact the author for the final version of the publication, or visit the DOI to the publisher's website.

- The final author version and the galley proof are versions of the publication after peer review.

- The final published version features the final layout of the paper including the volume, issue and page numbers.

Link to publication

\footnotetext{
General rights rights.

- You may freely distribute the URL identifying the publication in the public portal. please follow below link for the End User Agreement:

www.umlib.nl/taverne-license

Take down policy

If you believe that this document breaches copyright please contact us at:

repository@maastrichtuniversity.nl

providing details and we will investigate your claim.
}

Copyright and moral rights for the publications made accessible in the public portal are retained by the authors and/or other copyright owners and it is a condition of accessing publications that users recognise and abide by the legal requirements associated with these

- Users may download and print one copy of any publication from the public portal for the purpose of private study or research.

- You may not further distribute the material or use it for any profit-making activity or commercial gain

If the publication is distributed under the terms of Article $25 \mathrm{fa}$ of the Dutch Copyright Act, indicated by the "Taverne" license above, 
Stan van Hoesel

An overview of Stackelberg pricing in networks

$\mathrm{RM} / 06 / 043$

JEL code: C61

\section{METEबrR}

Maastricht research school of Economics of TEchnology and ORganizations

Universiteit Maastricht

Faculty of Economics and Business Administration

P.O. Box 616

NL - 6200 MD Maastricht

phone : ++31 433883830

fax $\quad$ : ++31433884873 



\title{
An overview of Stackelberg pricing in networks
}

\author{
Stan van Hoesel *
}

October 6, 2006

\begin{abstract}
The Stackelberg pricing problem has two levels of decision making: tariff setting by an operator, and then selection of the cheapest alternative by customers. In the network version, an operator determines tariffs on a subset of the arcs that he owns. Customers, who wish to connect two vertices with a path of a certain capacity, select the cheapest path. The revenue for the operator is determined by the tariff and the amount of usage of his arcs. The most natural model for the problem is a (bilinear) bilevel program, where the upper level problem is the pricing problem of the operator, and the lower level problem is a shortest path problem for each of the customers.

This paper contains a compilation of theoretical and algorithmic results on the network Stackelberg pricing problem. The description of the theory and algorithms is generally informal and intuitive. We redefine the underlying network of the problem, to obtain a compact representation. Then we describe a basic branch-and-bound enumeration procedure. Both concepts are used for complexity issues and for the development of algorithms: establishing NP-hardness, approximability, special cases solvable in polynomial time, and an efficient exact branch-and-bound algorithm.
\end{abstract}

\section{Introduction}

Combinatorial optimization problems on networks generally involve costs on the arcs. The issue is to find the cheapest subset structure of the arcs such as a path, a tree, or a matching. The decisions to be made are which arcs to include in the structure. In the problems discussed here, also the costs (tariffs) of a given subset of the arcs are to be determined. This introduces two levels of decisions. At the top level the tariffs of some arcs are determined by an operator or agent, the leader. Once this is done, at the bottom

${ }^{*}$ Faculty of Economics and Business Administration, University Maastricht, P.O. Box 616, 6200 MD Maastricht, The Netherlands, Email: s.vanhoesel@ke.unimaas.nl 
level, customers must decide which edges they use for their optimal structure. This set-up is a Stackelberg game. The customer's structure here, is a path between two specified vertices. The game for the leader is then to determine prices of the arcs controlled by him, such that the collected revenues on the shortest paths of the customers are as high as possible.

Applications with a natural network structure are found in different transportation sectors: toll optimization on roads such as the French highway system, see (Dewez 2004; Labbé, Marcotte, and Savard 1998), but also the German truck toll system; long-distance freight transportation overseas, see (Brotcorne et al. 2000), passenger transportation in trains, and finally information transportation in telecom networks, see (Basar and Srikant 2002). Note that an essential ingredient is that the customers must have alternatives, either the market should be oligopolistic or there should be different alternatives, such as the choice between cargo transportation with trains or trucks.

The problem is most naturally formulated as a bilinear bilevel program, see section 2, An integer linear program is described in (Labbé, Marcotte, and Savard 1998). This ILP is, however, not necessary in the description of techniques, and therefore we do not incorporate it. The shortest path graph (SPG) is introduced in section 3. This graph, introduced in (Bouhtou et al. 2002), reduces the size of the original network. It is also as a tool in solution methods and complexity proofs. With the SPG, we develop a basic branch-andbound scheme in section 4. In section 5. we describe a series of complexity results such as NP-hardness proofs, (in)approximability, and polynomial-time solvability. In section 6] a series of variants and extensions is described. This section contains some interesting open problems.

\section{Problem definition and model}

Consider a network represented by a directed graph $G=(N, A)$ with nodes $N$ and arcs $A$. The arc set $A$ is partitioned into two sets: the tariff $\operatorname{arcs} T$, and the fixed cost arcs $F$. The tariff arcs belong to the leader in the network and incur a tariff (to be determined by the leader) for routing a unit of a client's demand. The fixed arcs are owned by other agents in the network, whose tariffs are known a priori. The tariffs on the arcs of $T$ are determined such that the total revenue of the leader is maximized. Both the tariffs and the fixed costs are assumed to be nonnegative. The clients on the network route their demands from source to destination according to the shortest path with respect to total cost, where the total cost of a path is defined as the sum of all the tariffs and fixed costs on the arcs of the path. Whenever the client has a choice among multiple shortest paths with the same total cost but with different revenues for the leader, we suppose the client takes the shortest path that is most profitable to the leader.

We denote by $c_{a}$ the cost of routing a unit demand on a fixed cost arc $a \in F$, and by $t_{a}$ 
the cost of routing a unit demand on a tariff arc $a \in T$. The commodities are denoted by the set $K$. The demand of a commodity $k \in K$ is given by $d_{k}$. The source and destination of commodity $k$ are given by the pair $\left(s_{k}, t_{k}\right)$. The set of paths that connect $s_{k}$ and $t_{k}$ is given by $P_{k}$. For each path $p \in P_{k}$ we introduce $T_{p}$ for its set of tariff arcs, and $F_{p}$ for its set of fixed cost arcs. Furthermore, the cost of routing a unit demand on $p$ is denoted by its length $l_{p}(t)$, which is a function of the tariffs $t$. The length of $p$ is determined by the sum of the costs on the fixed arcs of the path, denoted by $c_{p}$, and the costs on the tariff arcs of the path, represented by $\pi_{p}(t)$. Thus, $l_{p}(t)=c_{p}+\pi_{p}(t)$, where $c_{p}=\sum_{a \in F_{p}} c_{a}$, and $\pi_{p}(t)=\sum_{a \in T_{p}} t_{a}$. Note that our model implicitly incorporates arcs with both fixed and tariff costs since we can divide such an arc $a$ with cost $c_{a}$ and tariff $t_{a}$ into two consecutive arcs: an arc with fixed $\operatorname{cost} c_{a}$ and an arc with tariff $t_{a}$.

To ensure that the problem is bounded, we assume that for each commodity there is an upper bound on the amount the customer is willing to pay, or there exists a path from source to destination which uses only fixed cost arcs.

The following formulation of the arc pricing problem is a direct translation of the above description.

$$
\begin{array}{ll}
\max _{t \geq 0} & \sum_{k \in K} d_{k} \pi_{p_{k}^{*}}(t) \\
\text { s.t. } & p_{k}^{*}=\arg \min _{p \in P_{k}} l_{p}(t) \quad \forall k \in K
\end{array}
$$

The formulation given by (11) is a bilevel problem where at the upper level the leader strives to maximize his revenue, while at the lower level the clients (followers) seek to minimize the cost of routing their demands. Notice that the bilevel program given by (II) is not polynomial in its input data, since the set of all possible paths for each client $k \in K$ may be exponential in the size of the problem instance.

Example 1 Consider the following network.

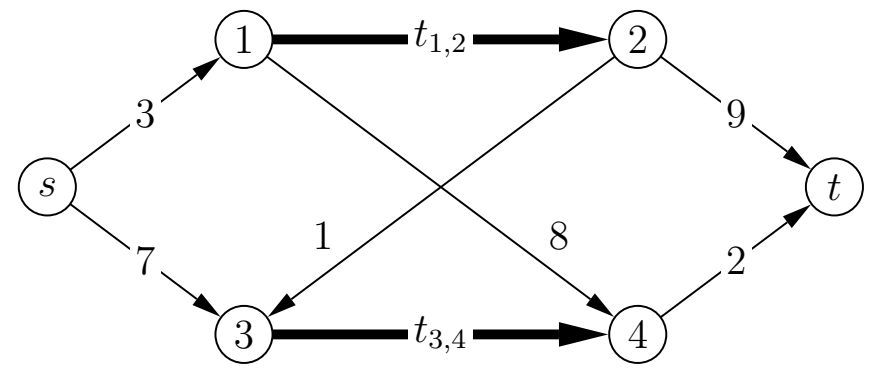

Figure 1: 1-commodity network with two tariff arcs. 


\begin{tabular}{l|rr|cc} 
Path & \multicolumn{2}{|c}{ Length } & \multicolumn{2}{c}{ Profit } \\
\hline s1234t & \multicolumn{2}{|c}{$6+t_{12}+t_{34}$} & $t_{12}$ & $+t_{34}$ \\
s12t & $9+t_{12}$ & $t_{12}$ & \\
s34t & 12 & $+t_{34}$ & & $t_{34}$ \\
s14t & 13 & & 0 &
\end{tabular}

In example 1 there are 4 paths connecting $s$ and $t$. Each of these paths has a fixed cost component and a tariff part. Though the path lengths are linear in the tariffs, the objective is not even continuous in the tariffs. This is illustrated in the example as follows. Let $t_{12}=0$, and start to increase $t_{34}$ from 0 to an arbitrary large value. Then up till value 3 the path $s 1234 t$ is optimal, and tariff and profit are equal, and after 3 the profit drops to 0 , since the path $\mathrm{s} 12 \mathrm{t}$ becomes the most attractive path for the customer. Note that the optimal solution for the leader is to set $t_{12}=3 t_{34}=3$, with a profit of 6 on the shortest path s1234t per unit demand.

(Labbé, Marcotte, and Savard 1998) considered the following arc oriented bilevel model. Let the vector $b^{k}$ be the demand/supply vector for each commodity where each element of the vector represents the demand/supply for a commodity at each node in the graph.

$$
\begin{array}{ll}
\max _{t \geq 0} & \sum_{k \in K} \sum_{a \in T} t_{a} x_{a}^{k} \\
\min _{x^{k} \geq 0} & \sum_{k \in K}\left\{\sum_{a \in T} t_{a} x_{a}^{k}+\sum_{a \in F} c_{a} x_{a}^{k}\right\} \\
\text { s.t. } & \sum_{a \in A_{i}^{+}} x_{a}^{k}-\sum_{a \in A_{i}^{-}} x_{a}^{k}=\left\{\begin{array}{rl}
d_{k} & i=s_{k} \\
-d_{k} & i=t_{k} \\
0 & \text { otherwise }
\end{array}\right.
\end{array}
$$

Here $A_{i}^{+}$is the set of arcs leaving $i$, and $A_{i}^{-}$is the set of arcs entering $i$. In this bilevel model, $x^{k} \in \mathbb{R}^{|A|}$ represents the flow on the arcs, in vector notation of commodity $k$. Furthermore, $A$ represents the node-arc incidence matrix of the network. This model is a bilinear bilevel program, since the upper level is linear in the tariff variables and the lower level is linear in the arc choice variables. Clearly, the formulation is not linear in the combination of these variables.

Formulation 2 has been used in (Brotcorne et al. 2000) and (Brotcorne et al. 2001) for the development of primal-dual heuristics in case of a single-commodity and multiple commodities, respectively. (Labbé, Marcotte, and Savard 1998) developed an integer linear programming formulation from the bilevel program as follows. The lower level problem is a set of shortest path problems each of which can be described as the linear program given in the lower level problem of 2. The optimal solution can now be characterized using duality theory: add the dual and set the two objectives of dual and primal equal to one 
another. Finally, the constraints and objective contain products of tariff and design variables of the arcs. These must be linearized by introducing new variables $t_{a}^{k} a \in T, k \in K$ for the products and appropriate constraints. This roughly triples the number of variables and constraints to $O(|K||T||V|)$. See (Labbé, Marcotte, and Savard 1998) for a complete description of the resulting model.

\section{The Shortest Path Graph}

If a client selects a shortest path, say $p$, then the subpaths of $p$ are also shortest paths. This holds specifically for the subpaths between two consecutive tariff arcs. Consider two such $\operatorname{arcs} a_{1}=\left(i_{1}, j_{1}\right) \in T$ and $a_{2}=\left(i_{2}, j_{2}\right) \in T$. Then the subpath between $j_{1}$ and $i_{2}$ is a shortest path that contains only fixed arcs.

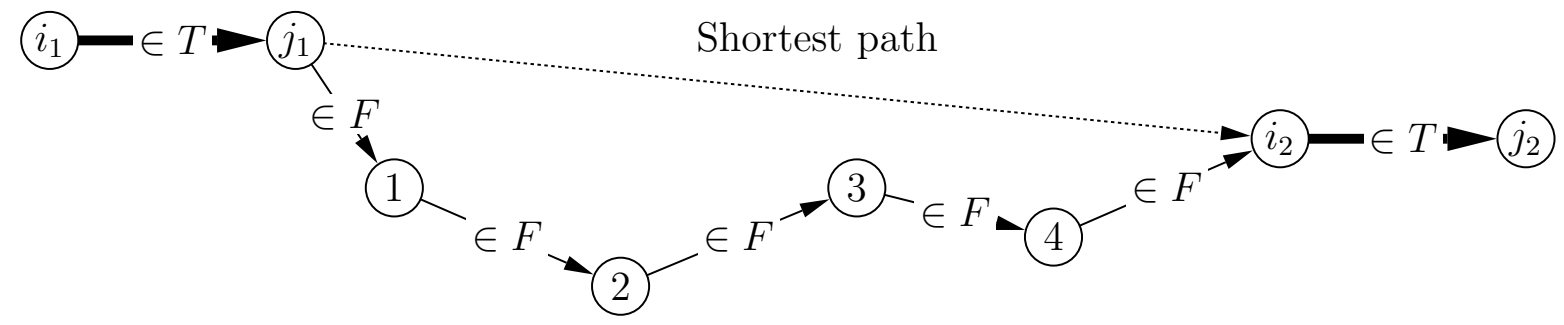

Figure 2: Shortest path of fixed cost arcs between $j_{1}$ and $i_{2}$.

Thus, we can restrict the client's choice to paths $p$ of the following structure:

$$
p=\left\{s p_{1}, a_{1}, s p_{2}, a_{2}, \ldots, s p_{k}, a_{k}, s p_{k+1}\right\}
$$

where $s p_{i}, i \in\{1, \ldots, k+1\}$ are shortest subpaths using only fixed cost arcs from a tariff arc $a_{i}$ to a tariff arc $a_{i+1}$ on the path. Since these subpaths are part of the subgraph using the arcs from $F$, their length can be computed without determining the tariffs. We can therefore construct a new graph model, in which this is actually done: the shortest path graph.

We will define this graph model for a single customer first. Consider the original graph $G=(N, A)$ with the tariff arcs in $T \subseteq A$. For a client with a demand $d$ from $s$ to $t$, we define the graph $G^{*}=\left(N^{*}, A^{*}\right)$ and the tariff arcs $T^{*} \subseteq A^{*}$. In this graph, the tariff arcs are copied from $G$ as a matching. So, arcs with a common vertex are separated. Next, we construct the following fixed cost arcs. For two tariff arcs $a_{1}=\left(i_{1}, j_{1}\right)$ and $a_{2}=\left(i_{2}, j_{2}\right)$ we connect $j_{1}$ with $i_{2}$, if there is a path in $G$ that uses fixed arcs only. Similarly, we connect $j_{2}$ with $i_{1}$. From the source $s$ we construct arcs to all the tail nodes of the tariff arcs, and from all the head nodes we construct an arc to the destination $t$, again only if paths exist 
using only fixed arcs in $G$. Any fixed arc in $A^{*}$ has a cost equal to the length of the shortest path between its end vertices in $G$, using only fixed cost arcs in $G$. The new network is the shortest path graph $(\mathrm{SPG})$.
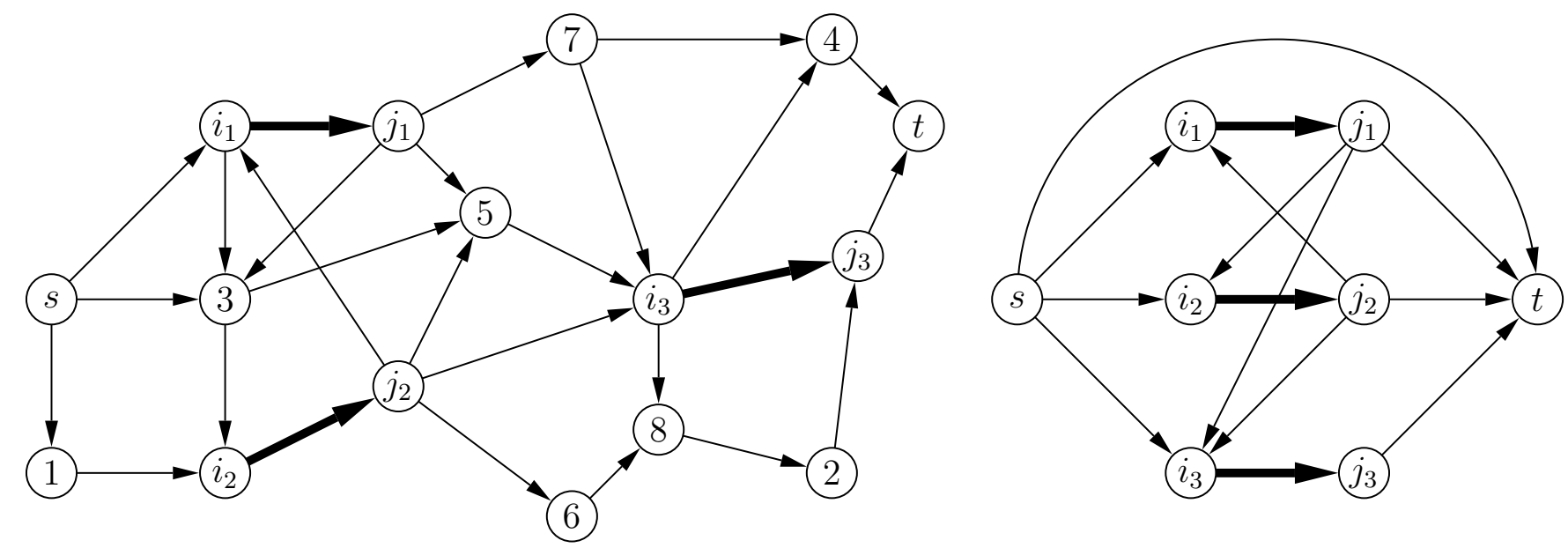

Figure 3: Shortest path graph.

Example 2 Figure 3 shows the SPG of a network containing three tariff arcs for a customer with demand from $s$ to $t$. The tariff arcs are given by the bold arcs $\left(i_{1}, j_{1}\right),\left(i_{2}, j_{2}\right)$ and $\left(i_{3}, j_{3}\right)$. All other arcs are representations of the shortest path using only fixed cost arcs between each node. The cost of the arc is the cost of the corresponding shortest path in the original network between the two nodes. The shortest path graph need not contain all possible arcs: if there is no path between two tariff arcs, then the connecting arc is missing in the SPG is also missing. In the example the $\operatorname{arcs}\left(j_{3}, i_{1}\right)$ and $\left(j_{3}, i_{2}\right)$ are missing.

In case of multiple customers, we create an SPG for each of them. The inner graph (consisting of the end vertices of the tariff arcs, and the arcs between them) is equal for all customers and hence needs to be determined only once. Additional shortest path calculations are necessary only for the arcs leaving the source and/or entering the target of each customer. The shortest path graph model is equivalent to the original graph in the sense that both have an optimal solution of the same value: each path in the original graph is represented by a path in the SPG which is at least as good. Alternatively, a path in the SPG, has exactly the same fixed costs as the shortest path in the original graph connecting the tariff arcs of the first in the same order, and thus it contains the same tariff arcs.

We can further reduce the SPG by removing arcs that will not be taken for any set of tariffs. This is done by use of (path) dominance criteria. Here, nonnegativity of the tariffs is vital. 
Definition 1 If, for any set of tariffs, the path $p$ is at least as short as path $q$, then path $p$ dominates path $q$.

The following proposition allows us to eliminate dominated paths. Recall that $T_{p}$ is the set of tariff arcs from path $p$, and that $c_{p}$ is the total cost of the fixed arcs from $p$.

Proposition 1 Consider two paths $p$ and $q$. If $T_{p} \subseteq T_{q}$ and $c_{q} \geq c_{p}$, then path $p$ dominates path $q$ for all tariffs.

By the nonnegativity of the tariffs and $T_{p} \subseteq T_{q}$, the total tariff on $p$ is at most the total tariff on $q$. Since this is also the case for the total fixed costs, $q$ will never be shorter than $p$. Thus, $q$ need never be selected by the customer.

Example 3 An instance where dominance of paths occurs is given in figure 4. The tariff arcs are the $\operatorname{arcs}\left(i_{1}, j_{1}\right)$, and $\left(i_{2}, j_{2}\right)$. The leader is dealing with one client who wants to route his demand from node $s$ to node $t$. For this graph, the path $\left\{s, i_{1}, j_{1}, i_{2}, j_{2}, t\right\}$ is dominated by the path $\left\{s, i_{1}, j_{1}, t\right\}$, since the arc $\left(j_{1}, t\right)$ has length 4 and the path $\left\{j_{1}, i_{2}, j_{2}, t\right\}$ has length at least 5 . In fact, the arc $\left(j_{1}, i_{2}\right)$ is never used and can therefore be removed

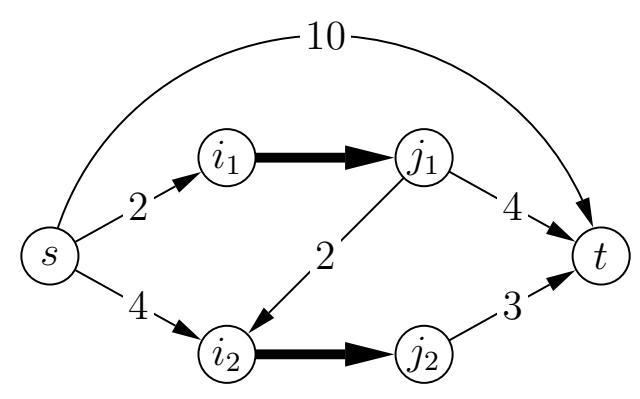

Figure 4: Path dominance.

An arc can be removed from the network, if every path using it is dominated by a path not using it. This may happen under various circumstances mentioned in (Bouhtou et al. 2002). We will restrict ourselves to a few straightforward ideas.

In principal, an arc can be removed if the fixed costs to reach it from $s$, or to leave it to $t$ are large enough. Thus, in example 5 , arc $\left(i_{3}, j_{3}\right)$ can be removed since reaching it costs at least 10 and leaving it costs 2 . So, the fixed costs for using $\left(i_{3}, j_{3}\right)$ are at least 12 , which is more than the cost of moving from $s$ to $t$ directly: 11. The arc $\left(j_{1}, i_{2}\right)$ can be removed, since the fixed costs of moving directly to $t$ are 4 , and leaving $j_{1}$ through $\left(j_{1}, i_{2}\right)$ has fixed costs of at least 6 . Similarly, arc $\left(j_{2}, i_{1}\right)$ can be removed. 

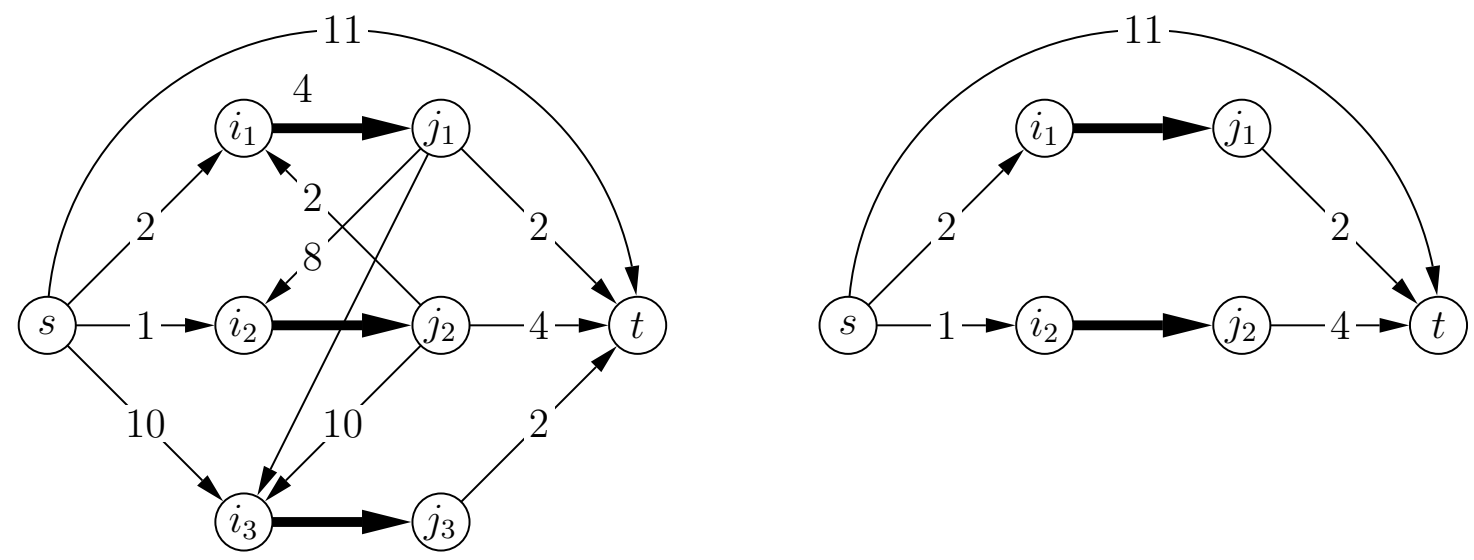

Figure 5: Arc removal.

In the SPG, the maximum number of paths for a client $k \in K$ is bounded by $e|T|$ !, the number of ordered subsets of the tariff arcs. This number is reached in a complete SPG. The number of undominated paths in a network is bounded by the number of possible subsets of $T$, i.e. by $2^{|T|}$ : If two paths $p$ and $q$ have an identical set of tariff arcs, then the undominated path is the path with smallest fixed cost. Figure 6] shows that this number of undominated paths can be reached for $|T|=4$, with an easy extension to arbitrary $|T|$.

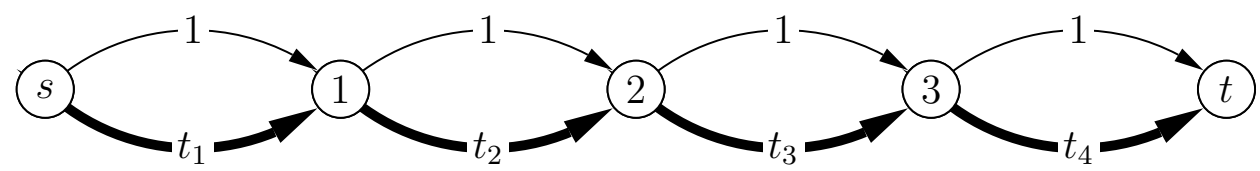

Figure 6: Network with 4 tariff arcs, and $2^{4}$ undominated paths.

\section{A basic Branch-and-Bound scheme}

In this section we describe a branch-and-bound algorithm for the pricing problem. This algorithm consists of two steps. In the first step we generate for each client his shortest path graph, and we enumerate all feasible undominated paths with their fixed costs and tariffs. In step two we solve the problem to optimality by branching and bounding on the paths.

Denote the clients by the set $K$ and the set of paths a client $k \in K$ can take by $P_{k}$. The reduction methods applied to the shortest path graph model allow us to determine the set of relevant paths for each customer. We suppose that $P_{k}$ is reduced to contain the relevant paths only. Recall that the linear function $l_{p}(t)=c_{p}+\pi_{p}(t)$ denotes the cost of a path $p$ as a function of all tariff values. Let $p_{k}^{l}$ be the path for client $k \in K$ with the smallest fixed cost, i.e., $p_{k}^{l}=\arg \min _{p \in P_{k}} c_{p}$ and $p_{k}^{u}$ the path with the largest fixed cost, i.e., $p_{k}^{u}=\arg \max _{p \in P_{k}} c_{p}$. Note that $p_{k}^{u}$ has no revenues for the leader, since it denotes the 
path with fixed cost arcs only. Clearly, $c_{p_{k}^{u}}-c_{p_{k}^{l}}$ is an upper bound on the revenues that can be generated from client $k$. This is an important measure in the branch and bound algorithm.

\subsection{Branching}

In each node of the branch and bound tree, we select a client, and we create a branch for each of the relevant paths of the client. The selection method of the clients is based on the upper bound $c_{p_{k}^{u}}-c_{p_{k}^{l}}$ on the revenue generated by each client for the leader: the client for which this upper bound is highest, is selected first. Next, we walk through the search tree in a depth-first manner.

\subsection{Bounding}

Due to our branching rules, in each node of the branch and bound tree for some clients the path taken in the solution is fixed, whereas for other clients this choice is still to be made. In each node, we denote by the set $K_{f} \subseteq K$ the set of clients for which we have fixed the path taken in the solution. Suppose that for any client $k \in K_{f}$, we have fixed the path $p_{k}^{*}$. We can find the optimal, revenue maximizing tariffs for the problem restricted to the clients in $K_{f}$ by solving the following linear problem.

$$
\begin{array}{lll}
\max & \sum_{k \in K_{f}} d_{k} \pi_{p_{k}^{*}}(t) & \\
\text { s.t. } & l_{p}(t) \geq l_{p_{k}^{*}}(t) & \forall k \in K_{f}, \forall p \in P_{k} \\
& t_{a} \geq 0 & \forall a \in T
\end{array}
$$

The linear program described in (4) forces the path $p_{k}^{*}$ to be the shortest path in $P_{k}$, while maximizing the leader's revenue.

We generate lower bounds in each node of the branch and bound tree by computing a feasible solution. Such a feasible solution can be created by solving (4) and then adding the revenues from the tariffs of (4) for the clients in $K \backslash K_{f}$.

Example 4 Consider a problem with 4 customers, with demand and list of undominated paths as follows:

\begin{tabular}{|lll|lll|ll|lll|}
\multicolumn{2}{c}{$d_{1}=20$} & \multicolumn{3}{c}{$d_{2}=10$} & \multicolumn{1}{c|}{$d_{3}=15$} & \multicolumn{2}{c|}{$d_{4}=5$} \\
\hline 1 & $+t_{1}$ & $+t_{2}$ & 2 & $+t_{1}$ & $+t_{2}$ & 3 & $+t_{1}$ & 2 & $+t_{12}$ & \\
3 & & $+t_{2}$ & 4 & $+t_{1}$ & & 5 & & 4 & & $+t_{12}$ \\
5 & & & 5 & & $+t_{2}$ & & & 7 & & \\
& & & & & & & & & & \\
\hline
\end{tabular}


The lower and upper bounding are as follows. Suppose that the subproblem to be solved is the one where client 1 has the second path as shortest path $\left(\right.$ cost $\left.3+t_{2}\right)$, and client 2 has the first path as shortest path (cost $\left.2+t_{1}+t_{2}\right)$. Then the following LP has to be solved:

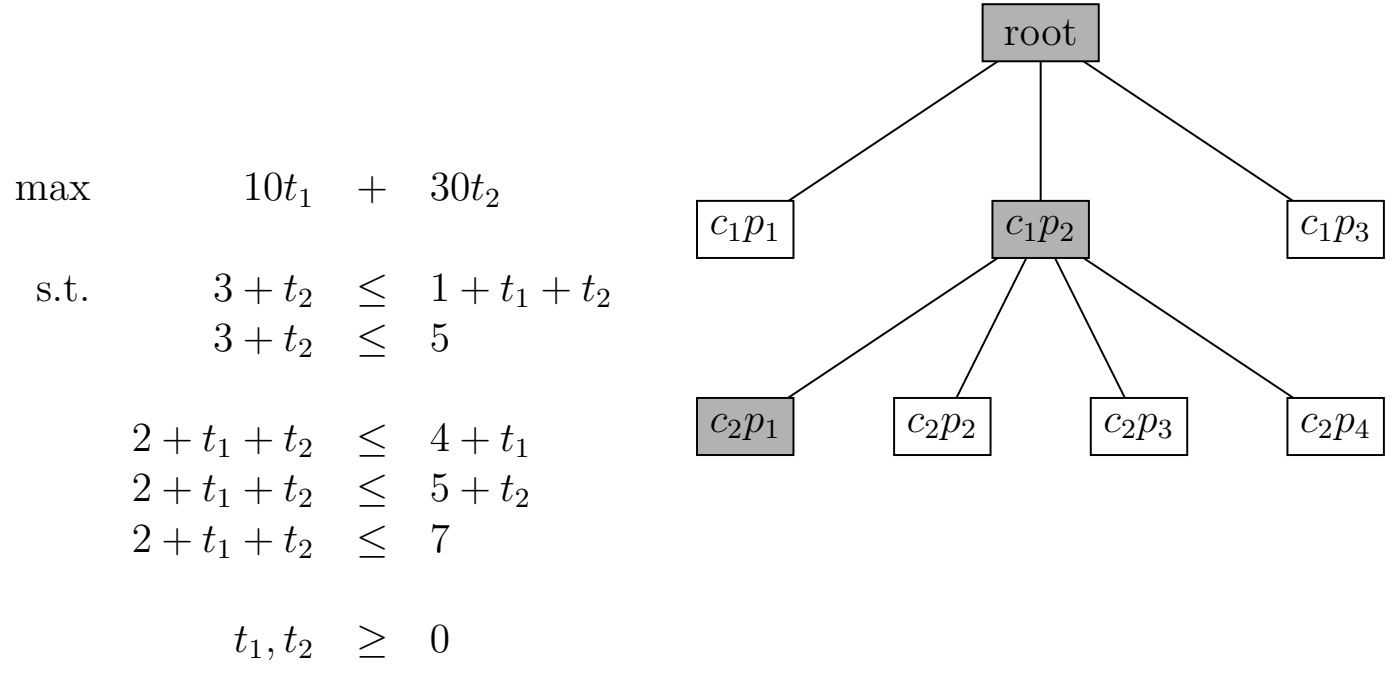

Figure 7: Subproblem with LP.

Note that the subproblems define a linear program to find a lower bound (feasible solution). In the problem above the LP generates the optimal solution $t_{1}=3, t_{2}=2$ with value 90 . The upper bound is now computed by taking the worst scenario: the gap between the fixed costs of the best path and the fixed cost path, multiplied with the demand of each of the remaining clients. In this case the upper bound is $15 * 2+5 * 5=55$ higher than the lower bound. Note that the lower bound is easily increased by taking the contribution of clients 3 and 4 into account. In Bouhtou et al. (Bouhtou et al. 2002) the upper and lower bounds are strengthened furthermore.

For a client $k \in K$ an upper bound for the unit demand revenue generated by that client is given by $c_{p_{k}^{u}}-c_{p_{k}^{l}}$. As is shown by Labbé et al. (Labbé, Marcotte, and Savard 1998), this upper bound is not necessarily reached. Even the upper bound on the cost of the path, $c_{p_{k}^{u}}$, is not tight. This is shown by the example given in figure 8 . For a single client with a unit demand from node 1 to node 4 , the optimal tarification scheme is to set the tariffs on the tariff arcs to $t_{1}=t_{2}=2$. Hence, the cost of the path taken by the client is 6 , yielding a revenue of 4 for the leader. The upper bound on the cost of the path is however 7 , while the upper bound on the revenue is given by $7-2=5$.

(Roch, Savard, and Marcotte 2005) give an example which shows that the relative gap can be logarithmic in the number of tariff arcs. In other words it can be arbitrarily large. 


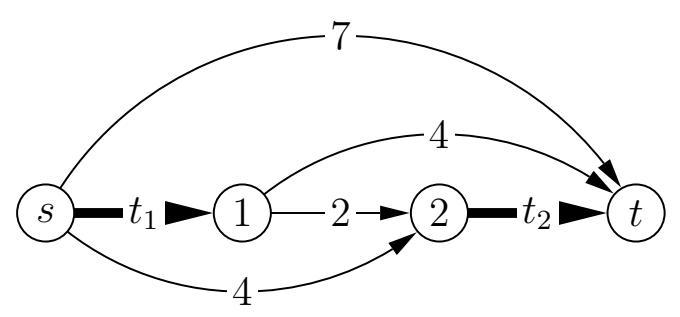

Figure 8: Example where the gap can not be used completely.

\section{Complexity}

\section{$5.1 \quad$ NP-hardness}

In (Labbé, Marcotte, and Savard 1998) the version of the problem with one customer and general (possibly negative) lower bounds on the tariffs, has been shown to be NP-hard. The proof uses a reduction from Hamiltonian path. Later, (Roch, Savard, and Marcotte 2005) proved that the one-customer problem is strongly NP-hard already, when all lower bounds equal 0. They use a reduction from 3-SAT, which we give below in a slightly modified version. Consider $n$ variables $x_{1}, \ldots, x_{n}$ and $m$ clauses $C_{1}, \ldots, C_{m}$. A clause is represented in the tariff network with the following construct.

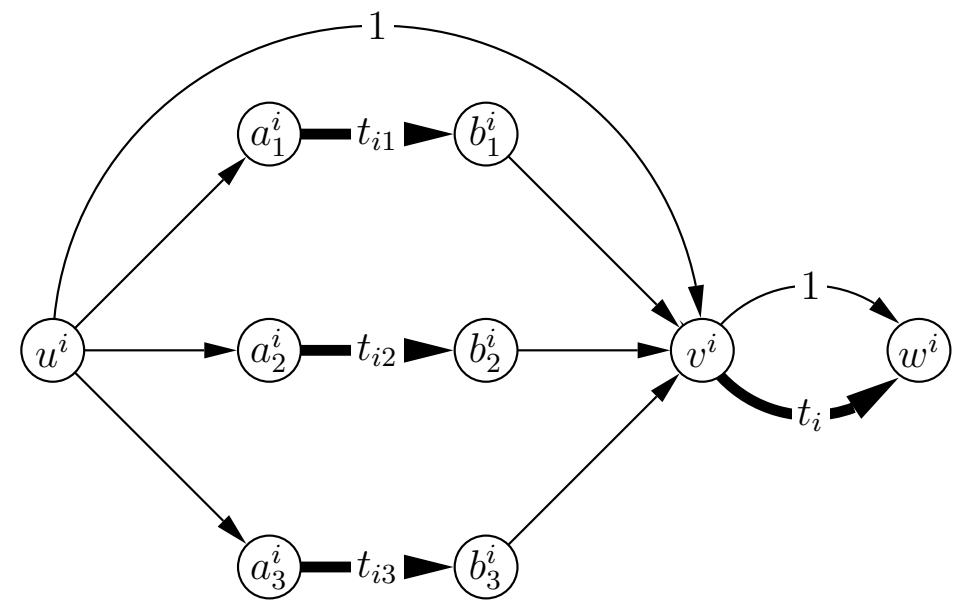

Figure 9: Clause $C_{i}$ for three arbitrary variables.

The left three toll arcs $\left(a_{j}^{i}, b_{j}^{i}\right)(j=1,2,3)$ with tariffs $t_{i j}$ correspond to the three variables in the clause. Besides the arc $\left(v^{i}, w^{i}\right)$ with tariff $t_{i}$, there are only fixed cost arcs, each having fixed cost 0 , except the arcs $\left(u^{i}, v^{i}\right)$ and $\left(v^{i}, w^{i}\right)$ with cost 1 .

The clauses are coupled by identifying the nodes $w^{i}$ and $u^{i+1}(i=1, \ldots, m-1)$. Finally, we add arcs with fixed cost $\frac{1}{2}$ between any pair of tariff arcs that corresponds to a variable 
and its negation. We connect the head vertex of the earlier clause arc to the tail vertex of the later clause arc. See the picture-be- $\overline{l o}^{-\frac{1}{2}}$ where-the dashed arcs are of this type. Finally, the source $s=1 t^{1}$ and the destination is $-1=w^{m}$.

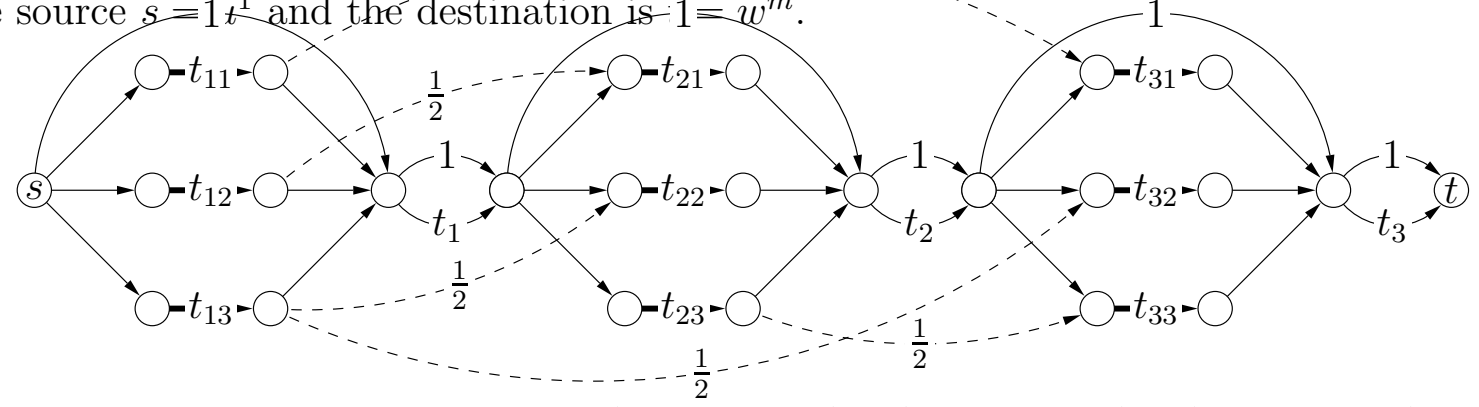

Figure 10: Network for formula $\left(x_{1} \vee x_{2} \vee \bar{x}_{3}\right) \wedge\left(\bar{x}_{2} \vee x_{3} \vee \bar{x}_{4}\right) \wedge\left(\bar{x}_{1} \vee x_{3} \vee x_{4}\right)$.

The idea is now to let the arcs of a selected (optimal) path correspond with a truth assignment of the variables in the 3-SAT problem.

Now, the tarification problem has a solution with value $2 m$ if and only if there is a truth assignment to the variables of the corresponding 3-SAT instance.

Note that the path $s=u^{1}, v^{1}, w^{1}, u^{2}, \ldots, w^{m-1}, u^{m}, v^{m}, w^{m}$, with fixed cost arcs has length $2 m$, and any path taking two tariff arcs in each clause construct has total fixed costs 0 . Moreover, each tariff arc can add at most 1 to the value of the problem, which bounds the value of any optimal solution to $2 m$.

First, suppose that an optimal path with value $2 m$ exists. This path will take two arcs with tariff 1 in each clause construct. Now, let the variables corresponding to the taken tariff arcs be set to true. Then no conflicts will arise: if both a variable and its negation are on the path (and thus set to true) in two different clauses, then there is a dashed arc connecting them. Since this arc is a shortcut of the path (note that we skip at least one arc with tariff 1 of type $\left.\left(v^{i}, w^{i}\right)\right)$, this contradicts the optimality of the path.

Second, suppose that the 3-SAT problem has a valid truth assignment. Set the tariffs on the variables in the clauses which are false to a value higher than $2 m$, and all other tariff arcs to value 1. Clearly, the dashed arcs are never used in an optimal path, since this would incorporate an arc with tariff higher than $2 m$, which is worse for the customer than the length of the fixed cost path. Finally, none of the arcs with fixed costs 1 will be used, since the alternative of one of the tariff arcs with tariff one is equally good for the customer and better for the operator. Thus, the optimal path will take two tariff arcs of value 1 in each clause construct, which leads to a total value of $2 \mathrm{~m}$.

\subsection{Approximation}

(Roch, Savard, and Marcotte 2005) give also an approximation algorithm for the problem with one customer, with a performance guarantee of $O(\log T)$. The idea of the algorithm 
is fairly easy. It successively tries to find paths with high tariff revenue. It starts with the best path $P$ possible: the one with the smallest amount of fixed costs. After computing the optimal tariffs for $P$, the tight fixed cost arcs are identified. The one with the smallest cost replaces the subpath in $P$ it connects. For the new path the procedure is repeated until all tariff arcs are removed.

A subroutine of this procedure is to find the best tariffs given that the path $P$ is optimal. Tariff arcs not in $P$ get sufficiently high tariffs, in order not to be a problem. For the arcs in $P$ a greedy algorithm does the job: in order of appearance in $P$ each arc gets a tariff as high as possible.

The analysis of the algorithm is the complicated part. The bound is tight for this particular algorithm, as shown by an example given in (Roch, Savard, and Marcotte 2005).

(Grigoriev et al. 2004) show that in the special case where the inner graph of the SPG contains only tariff $\operatorname{arcs} O(\log T)$ is worst-case even for multiple customers. They also prove that this problem is APX-hard.

\subsection{Polynomially solvable cases}

In (Labbé, Marcotte, and Savard 1998) many special cases that are polynomially solvable have been identified. One of them is the single-customer case, where the order of used tariff arcs is known. Another is the single tariff-arc problem. (van der Kraaij 2004) proves that even in the case of fixed charge costs this problem is polynomially solvable. We will concentrate here on the problem where the number of tariff arcs in not part of the input, i.e., bounded of fixed beforehand.

The bilevel program defined in (11) is shown to be equivalent to a set of linear programs. Consider the problem where we force for each client a specific relevant path to be shortest. Then, as illustrated in the section on Branch-and-bound, the determination of optimal tariffs, if feasible, is a linear program. Since, in the optimal solution, there is a set of shortest paths for the clients, we can consider any possible set of shortest paths and solve the corresponding LP. However, doing this directly does not result in a running time polynomial in the number of customers. We dig a little deeper in the structure of the constraints, to get the desired result.

For any client $k \in K$, consider two paths $p_{1}, p_{2} \in P_{k}$. If $p_{1}$ is to be the shortest of the two paths, the constraint $l_{p_{1}}\left(d_{k}\right) \leq l_{p_{2}}\left(d_{k}\right)$ must hold. Thus:

$$
c_{p_{1}}\left(d_{k}\right)+\pi_{p_{1}}\left(d_{k}\right) \leq c_{p_{2}}\left(d_{k}\right)+\pi_{p_{2}}\left(d_{k}\right) \Longleftrightarrow \pi_{p_{1}}\left(d_{k}\right)-\pi_{p_{2}}\left(d_{k}\right) \leq c_{p_{2}}\left(d_{k}\right)-c_{p_{1}}\left(d_{k}\right)
$$

This constraint is of the form: 


$$
\sum_{a \in T_{1}} t_{a}-\sum_{a \in T_{2}} t_{a} \leq b^{k}\left(p_{1}, p_{2}\right)
$$

Here, $b^{k}\left(p_{1}, p_{2}\right)$ is a constant and $T_{1}$ and $T_{2}$ are disjoint subsets of $T$. Note that $T_{1}$ contains the tariff arcs in $p_{1}$ not in $p_{2}$, and $T_{2}$ contains the tariff arcs in $p_{2}$ not in $p_{1}$. The constant $b^{k}\left(p_{1}, p_{2}\right)$ is referred to in the remainder as the switching value for the pair $\left(p_{1}, p_{2}\right)$ for a client $k \in K$. The number of different left-hand sides of $\left[\right.$ is $3^{\mid} T \mid$, since each variable can have coefficient only in $\{-1,0,1\}$. The number of switching-values per client is the number of different pairs of paths, and that is bounded by $(e T !)^{2}$.

The main idea is to collect all possible switching values $b_{r}$, and to order them: $(r \in$ $\{1, \ldots, R\})$. The next point is that we create our set of LPs as follows. For each disjoint pair of subsets of $T, T_{1}$ and $T_{2}$ we fix a consecutive pair of switching values with the index $r\left(T_{1}, T_{2}\right)$, and we add the following constraint to the LP:

$$
b_{r\left(T_{1}, T_{2}\right)-1} \leq \sum_{a \in T_{1}} t_{a}-\sum_{a \in T_{2}} t_{a} \leq b_{r\left(T_{1}, T_{2}\right)}
$$

It is not hard to show that the number of LPs that we can create is polynomial in the number of clients, but exponential in the number of tariff arcs. For details, see van Hoesel et al. 2003).

\section{Variants of the Stackelberg pricing problem}

\subsection{Special cases}

The structure of the network can be restricted in the sense that the tariff arcs meet certain properties. Two obvious properties are: the tariff arcs form a cut-set (Grigoriev et al. 2004), or the tariff arcs form a path (?). The first problem is not easier than the original problem: it is still NP-hard, for multiple customers and the best known approximation algorithm does not improve the one given in the previous section. The second problem is under recent investigation, with a slight modification: using multiple arcs in common the total tariff may be smaller than the sum of tariffs of individual arcs. It is not known whether this problem is NP-hard. Its application is found in toll systems with many entrances and exits, such as the French highway structure.

\subsection{Extensions}

Extensions can be defined in several ways. One way is to incorporate capacities on the arcs. This extension is quite hard to handle. For instance, the path-oriented ideas should 
be replaced with network oriented ideas. No research on this subject has been reported, as far as known to the author.

A second extension is the pricing mechanism. Instead of linear tariffs, these may be fixed charge, or even just increasing with demand. The $\mathrm{PhD}$ thesis of (van der Kraaij 2004) contains an analysis of the problem with different types of cost structures. It shows that the case with one tariff arc is polynomial for the fixed charge costs. Moreover, it shows how the branch-and-bound algorithm can be used.

A third extension is the incorporation of the design of the network. The following formulation of the tarification problem is a direct translation of the above description. Here, $P_{k}$ is the set of paths in the network $G=\left(V, F+T^{\prime}\right)$ where $T^{\prime}$ is the subset of $T$ of selected arcs, for which a cost $c_{a}$ is involved. In (Brotcorne et al. 2005) the problem is formulated and solved with heuristics and a specialized Lagrangean relaxation approach.

\subsection{Related problems}

In traffic congestion problems time can be considered as a price. Here, however, the time is dependent on the capacity usage, whereas in our case we have only a linear relation between capacity usage and price. Examples are routing of traffic flows, see (Roughgarden and Tardos 2000) and (Roughgarden 2001) and IP traffic engineering, see (Fortz and Thorup 2000). (Basar and Srikant 2002) treat traffic congestion problems explicitly as a Stackelberg game on networks.

\subsection{General Bilevel Programs}

The general linear-linear bilevel program has been shown to be $\mathcal{N} \mathcal{P}$-hard by Jeroslow (Jeroslow 1985). For a reference on bilevel programming, we refer the reader to Vicente and Calamai (Vicente and Calamai 1994) who have compiled an annotated bibliography on this subject containing more than one hundred references.

\section{Concluding remarks}

The standard Stackelberg pricing problem on networks is well-solved from a practical point of view, and also many important theoretical questions have been answered. Nevertheless, some interesting open problems remain: approximability for special cases within a constant factor, and cutting plane methods for the integer linear programming formulation of the problem. Branch-and-cut methods are currently developed by different groups. A preliminary study can be found in (Dewez 2004). 
For extensions of the problem, many of the results discussed here have no counterpart in the extensions. For instance, the capacitated case has no good algorithmic methods, and no results on approximability. This, of course, applies also for other network or combinatorial bilevel programs. In other words, the field is still rich of open interesting questions.

\section{References}

Basar, T., and R. Srikant. 2002. "A Stackelberg network game with a large number of followers." Journal of Optimization Theory and Applications 115 (3): 479-490.

Bouhtou, M., S. van Hoesel, A. F. van der Kraaij, and J.L. Lutton. 2002. "Linear Tarification in Multi-Commodity Telecommunications Networks." Meteor Research Memorandum, vol. RM02012.

Brotcorne, L., M. Labbé, P. Marcotte, and G. Savard. 2000. "A Bilevel Model and Solution Algorithm for a Freight Tariff-Setting Problem." Transportation Science 34 (3): 289-302.

—. 2001. "A bilevel model for toll optimization on a multicommodity transportation network." Transportation Science 35 (4): 345-358.

—. 2005. "Joint Design and Pricing on a Network." Working Paper.

Dewez, S. 2004. "On the Toll Setting Problem." Ph.D. diss., Université Libre de Bruxelles.

Fortz, B., and M. Thorup. 2000. "Internet Traffic Engineering by Optimizing OSPF Weights." Proc. 19th IEEE Conf. on Computer Communications (INFOCOM). 519528.

Grigoriev, A., S. van Hoesel, A. F. van der Kraaij, M. Uetz, and M. Bouhtou. 2004. "Pricing network edges to cross a river." Meteor Research Memorandum RM04009, no. To appear in Naval Research Logistics Quarterly.

Jeroslow, R.G. 1985. "The polynomial hierarchy and a simple model for competitive analysis." Mathematical Programming 32:146-164.

Labbé, M., P. Marcotte, and G. Savard. 1998. "A bilevel model of taxation and its application to optimal highway pricing." Management Science 44:1608-1622.

Roch, S., G. Savard, and P. Marcotte. 2005. "Design and Analysis of an approximation algorithm for Stackelberg network pricing." 46(1):57-67.

Roughgarden, T. 2001. "Designing networks for selfish users is hard." Proceedings of the 42nd Annual IEEE Symposium on the Foundations of Computer Science. 472-482.

Roughgarden, T., and Tardos. 2000. "How Bad is Selfish Routing?" Proceedings of the 41st Annual IEEE Symposium on the Foundations of Computer Science. 93-102.

van der Kraaij, A.F. 2004. "Pricing in Networks." Ph.D. diss., University Maastricht. 
van Hoesel, S., A. F. van der Kraaij, C. Mannino, G. Oriolo, and M. Bouhtou. 2003. "Polynomial cases of the tarification problem." Meteor Research Memorandum, vol. RM03053.

Vicente, L.N., and P. H. Calamai. 1994. "Bilevel and multilevel programming: A bibliography review." Journal of Global Optimization 5:291-306. 